Pacific Journal of Mathematics

TWISTING TO ALGEBRAICALLY SLICE KNOTS 


\section{TWISTING TO ALGEBRAICALLY SLICE KNOTS}

\section{STEVE J. KAPLAN}

It is shown that every knot with zero Arf invariant can be made algebraically slice by a $(-1,-1)$-twist.

Suppose $K$ is a knot in $S^{3}$. For each integer $k$, consider the homeomorphism $H_{k}: D^{2} \times I \rightarrow D^{2} \times I$ defined by $H\left(r e^{i \theta}, t\right)=\left(r e^{i(\theta+2 \pi k t)}, t\right)$. Orient $S^{3}$ by the right-hand rule.

DEFINITION. A knot $K^{\prime} \subset S^{3}$ is obtained from $K$ by a $(k, l)$-twist if there exists a smooth embedding $f: D^{2} \times I \rightarrow S^{3}$ preserving orientation such that: times;

(i) $K$ intersects $f\left(D^{2} \times\{0\}\right)$ transversely and algebraically $l$

(ii) $K \cap f\left(D^{2} \times I\right) \subset f\left(\left(\right.\right.$ int $\left.\left.D^{2}\right) \times I\right)$; and

(iii) $K^{\prime}=K-\left(K \cap f\left(D^{2} \times I\right)\right) \cup f H_{k} f^{-1}\left(K \cap f\left(D^{2} \times I\right)\right)$.

Example:
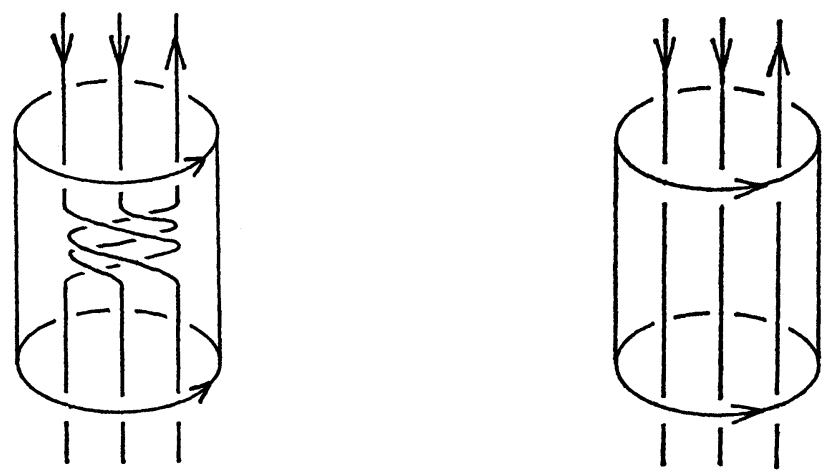

DIAGRAM 1

In [4], Akbulut and Kirby made the following three-tiered conjecture:

ConJECtURe 1. Suppose $K \subset S^{3}$ is a knot with zero Arf invariant. Then there exists a knot $K^{\prime}$ obtained from $K$ by a $(-1,-1)$-twist such that
A: $K^{\prime}$ is algebraically slice
B: $K^{\prime}$ is slice (or ribbon)
C: $K^{\prime}$ is the unknot.

Suppose $K$ is a knot with Arf invariant zero. If Conjecture 1B is valid for $K$, the homology 3-sphere obtained by surgery on $K$ with +1 framing must bound an acyclic manifold $W$ with $\pi_{1}(\partial W) \rightarrow$ 
$\pi_{1}(W)$ onto. A partial converse also holds. For a given $K$, if a manifold $W$ as above exists and a certain homotopy $C P^{2}$ is genuine, $K$ is concordant to a knot for which Conjecture $1 \mathrm{~B}$ is true [2]. If Conjecture $1 \mathrm{~B}$ were known for the $(2,7)$-torus knot it would be easy to construct a smooth, closed, simply connected, almost parallelizable 4-manifold with index and second Betti number equal to 16. This is considered unlikely.

Suppose $K$ and $K^{\prime}$ are knots such that $K^{\prime}$ is obtained from $K$ by a $(-k,-1)$-twist, It follows from a result of Tristram [5] that if $k \geqq 2$ the $k$-signatures of $K$ and $K^{\prime}$ coincide. The $k$-signatures need not be invariant under a $(-1,-1)$-twist. Akbulut [1] has provided an example of a knot $K$ for which Conjecture 1B is true such that $\sigma_{k}(K) \neq 0$ for every $k \geqq 2$. In light of this example it is not surprising that Conjecture $1 \mathrm{~A}$ is true. A proof is supplied below.

TheOREM. Suppose $K \subset S^{3}$ is a knot with Arf invariant zero. Then there exists a knot $K^{\prime}$ such that $K^{\prime}$ is obtained from $K$ by a $(-1,-1)$-twist and such that $K^{\prime}$ is algebraically slice.

REMARK. A. Casson has obtained the following related result: If $K$ is a knot with Arf invariant zero, there exist knots $K^{\prime}$ and $K^{\prime \prime}$ such that $K^{\prime}$ is concordant to $K, K^{\prime \prime}$ is obtained from $K^{\prime}$ by a $(-1,-1)$-twist, and the Alexander polynomial of $K^{\prime \prime}, \Delta(t)=1$. (A knot with $\Delta(t)=1$ must be algebraically slice.)

Proof of the Theorem. Let $F$ be a Seifert surface for $K$ and $a_{1}, b_{1}, \cdots, a_{n}, b_{n}$ a system of canonical curves for $F$. Since $\operatorname{Arf}(K)=0$, we may assume that the diagonal entry of the Seifert matrix arising from $a_{i}$ is even for each $i=1, \cdots, n$ (see [3]).

For each $i=1, \cdots, n$ be $c_{i}$ and $d_{i}$ be the cores of the handles pictured in Diagram 2:

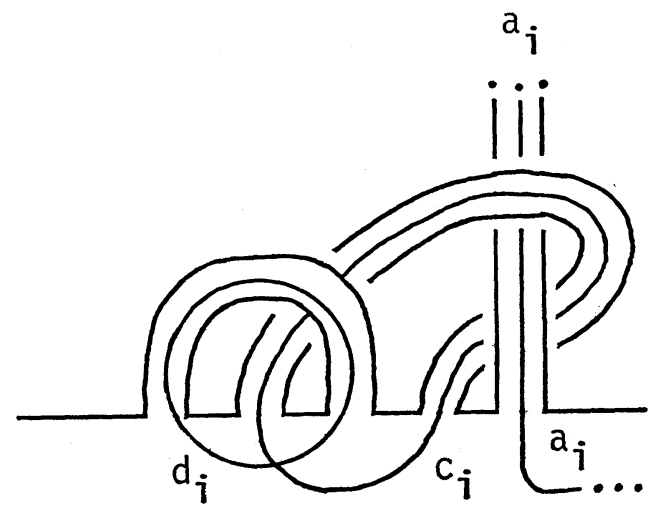

DIAGRAM 2 
Let $\bar{F}$ be the union of $F$ with these $2 n$ additional handles. Plainly $\partial \vec{F}$ is isotopic to $\partial F$. (By abuse of notation we write $\partial \bar{F}=K$.) The matrix describing the restriction of the Seifert form to the generators $a_{1}, \cdots, a_{n}, c_{1}, \cdots, c_{n}$ is $\left[\begin{array}{cc}A & I \\ I & 0\end{array}\right]$ where $A=\left(\alpha_{i j}\right)$ corresponds to the $a_{i}$. (Recall that the value of the Seifert form on the pair of curves $(x, y)$ of $\bar{F}$ is defined to be $l k\left(x, i^{*} y\right)$ where $i$ is a normal vector field to $\bar{F}$.) For each $i=1, \cdots, n$ define classes $\left[\alpha_{i}^{\prime}\right] \in H_{1}(\bar{F})$ by $\left[a_{i}^{\prime}\right]=\left[a_{i}\right]+\sum_{j=1}^{i}\left(1-\alpha_{i j}\right)\left[c_{j}\right]+\left(\alpha_{i i} / 2\right)\left[c_{i}\right]$. The class $\left[a_{i}^{\prime}\right]$ can be realized by a connected sum of $a_{i}$ with push-offs of the $c_{j}$. Thus there exist disjointly embedded curves $a_{1}^{\prime}, \cdots, a_{n}^{\prime}$ which represent the $\left[a_{i}^{\prime}\right]$ and are disjoint from the $c_{j}$. A simple calculation shows that the values of the Seifert form on $a_{1}^{\prime}, \cdots, a_{n}^{\prime}, c_{1}, \cdots, c_{n}$ are described by the matrix $\left[\begin{array}{ll}B & I \\ I & O\end{array}\right]$ where the diagonal entries of $B$ are +2 and the off-diagonal entries +1 .

Choose additional curves $b_{1}^{\prime}, \cdots, b_{n}^{\prime}, d_{1}^{\prime}, \cdots, d_{n}^{\prime}$ on $\bar{F}$ so that $\left\{a_{1}^{\prime}, b_{1}^{\prime}\right.$, $\left.\cdots, a_{n}^{\prime}, b_{n}^{\prime}, c_{1}, d_{1}^{\prime}, \cdots, c_{n}, d_{n}^{\prime}\right\}$ form a system of canonical curves and regard $\bar{F}$ as a disk with bands with these curves as cores. Let $l \subseteq \bar{F}$ be an arc whose intersection with $\partial \bar{F}$ is one of its endpoints and which misses each of the canonical curves (see Diagram 3). Consider the 2-disk $D \subset S^{3}$ pictured in Diagram 3 where the strands in the box $\begin{aligned} & l_{i} \\ & n_{i}\end{aligned}$ run parallel to an arc $l_{i} \subset S^{3}-\bar{F}$ and perform $n_{i}(\in Z)$ full twists about $l_{i}$. The (transverse) intersection of $D$ and $\bar{F}$ consists of the co-cores of the bands with cores $a_{1}^{\prime}, \cdots, a_{n}^{\prime}$ together with $l$. We will show that for certain choices of $\left(l_{i}, n_{i}\right)$, the knot $K^{\prime}$ obtained from $K$ by a $(-1,-1)$-twist along $D$ is algebraically slice.

A portion of the knot $K^{\prime}$ and a portion of a genus $3 n$ Seifert
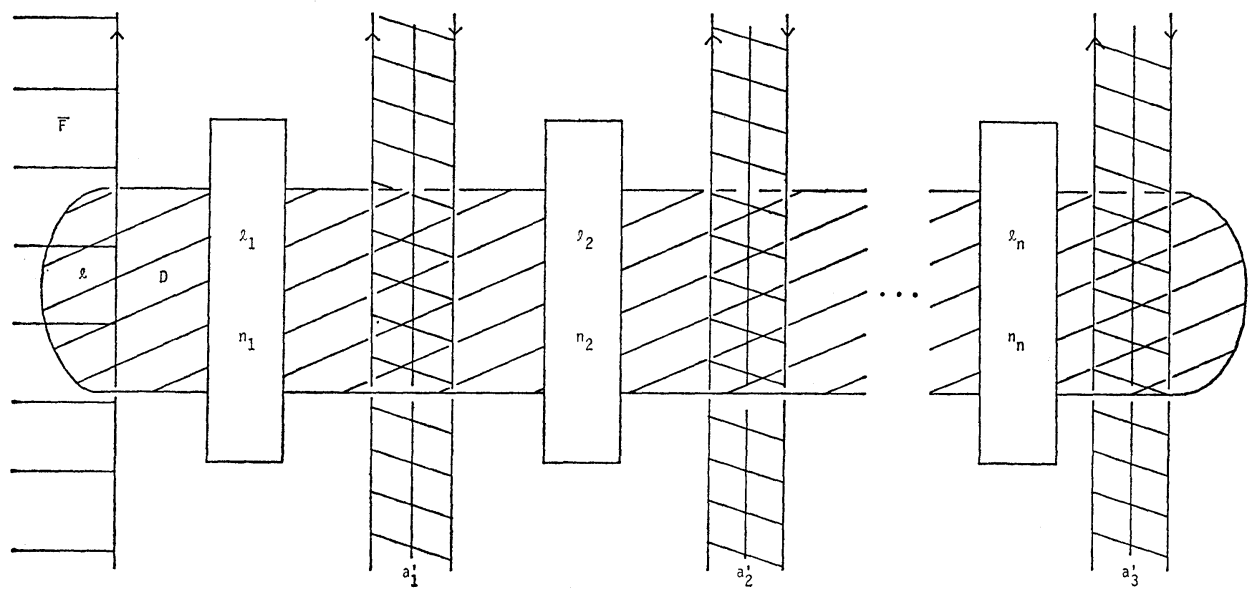

DiAgRAM 3 


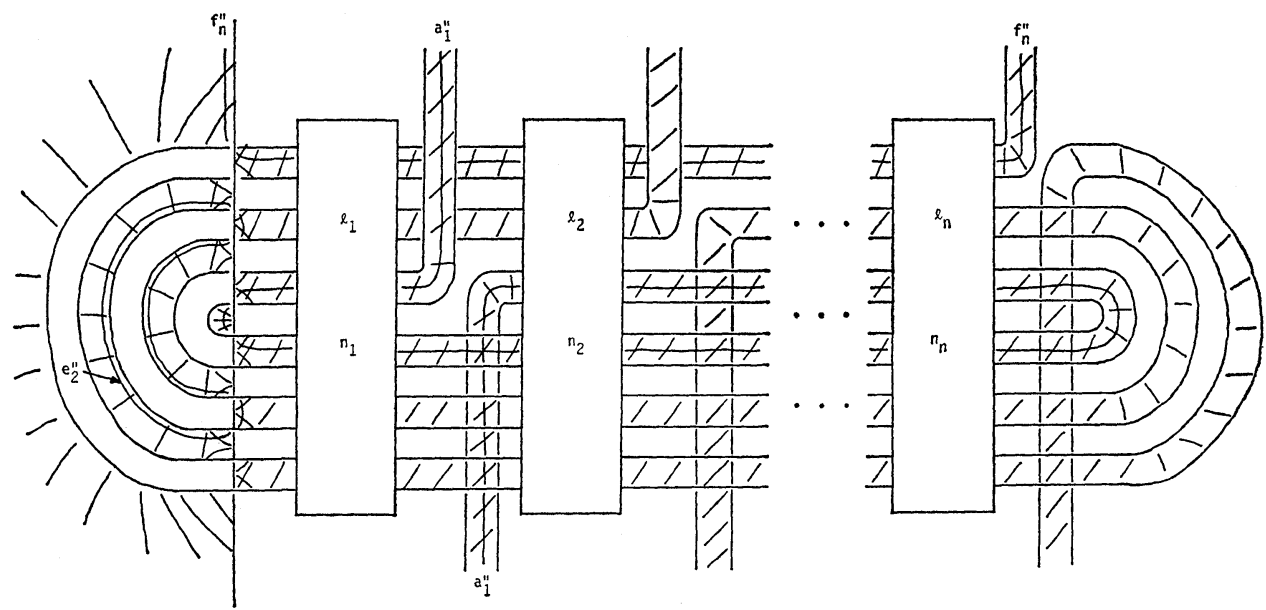

DIAGRaM 4

surface $G$ for $K^{\prime}$ are pictured in Diagram 4. ( $G$ is orientable because the $n_{i}$ are integral.) The part of $\left(G, K^{\prime}\right)$ absent from Diagram 4 agrees identically with the part of $(\bar{F}, K)$ not in Diagram 3. Thus the curves $b_{i}^{\prime}, c_{i}, d_{i}^{\prime}, i=1, \cdots, n$ represent generators of $H_{1}(G)$. Define $b_{1}^{\prime \prime}, c_{i}^{\prime \prime}, d_{i}^{\prime \prime}$ resp. to be the same curves regarded as curves on $G$.

Additional curves $a_{i}^{\prime \prime}, e_{i}^{\prime \prime}, f_{i}^{\prime \prime} i=1, \cdots, n$ completing a symplactic basis are shown in Diagrams 4 and 5 . The curve $a_{i}^{\prime \prime}$ is obtained as the twist of $a_{i}^{\prime}$ along $D$ except near $l$ where $a_{i}^{\prime \prime}$ follows a sheet of $G$ through two left half-twists. The curves $e_{1}^{\prime \prime}, \cdots, e_{n}^{\prime \prime}$ generate the homology of the part of $G$ near $l$ and are shown in Diagram 5. The curve $f_{i}^{\prime \prime}$ is dual (in $H_{1}(G)$ ) to $e_{i}^{\prime \prime}$. It is obtained as the union of an arc which runs once along $l_{j}, 1 \leqq j \leqq i$ (and is shown in Diagrams 4 and 5) with an arc outside Diagram 4 which misses each of the other canonical curves.

Suppose that choices of $\left(l_{i}, n_{i}\right)$ have been made so that the entries of the Seifert matrix of $G$ are defined. We shall later modify these choices. Let $\Lambda \subset H_{1}(G)$ be the subgroup generated by the classes of $a_{1}^{\prime \prime}, \cdots, a_{n}^{\prime \prime}, c_{1}^{\prime \prime} \# e_{1}^{\prime \prime}, \cdots, c_{n}^{\prime \prime} \# e_{n}^{\prime \prime}$. (The connected sums are taken along $G$.) Let $\Gamma \subset H_{1}(G)$ be the subgroup generated by the elements of $\Lambda$ together with the classes of $f_{1}^{\prime \prime} \#\left(-d_{i}^{\prime \prime}\right), \cdots, f_{n}^{\prime \prime} \#\left(-d_{n}^{\prime \prime}\right)$. It follows from the calculations above and the nature of the linking in Diagram 4 that the intersection form of $H_{1}(G)$ vanishes on $\Gamma$ and the Seifert form vanishes on $\Lambda$.

Let $C_{1}, \cdots, C_{n}$ be disjoint curves in $S^{3}-G$ satisfying

(i) $l k\left(C_{i}, x\right)=-l k\left(f_{i}^{\prime \prime} \#\left(-d_{i}^{\prime \prime}\right), x\right)$ for each generator $x$ of $\Lambda$, and

(ii) $l k\left(C_{i}, C_{j}\right)=-l k\left(f_{i}^{\prime \prime} \#\left(-d_{i}^{\prime \prime}\right), f_{j}^{\prime \prime} \#\left(-d_{j}^{\prime \prime}\right)\right)$ for each $j \neq i$.

Replace $l_{i}$ by $l_{i} \# C_{i} i=1, \cdots, n$. The entries of the Seifert matrix on $\Gamma$ are now zeros except possibly for the diagonal entries corresponding to the $f_{i}^{\prime \prime} \#\left(-d_{i}^{\prime \prime}\right)$. Clearly, these can be made zero by 


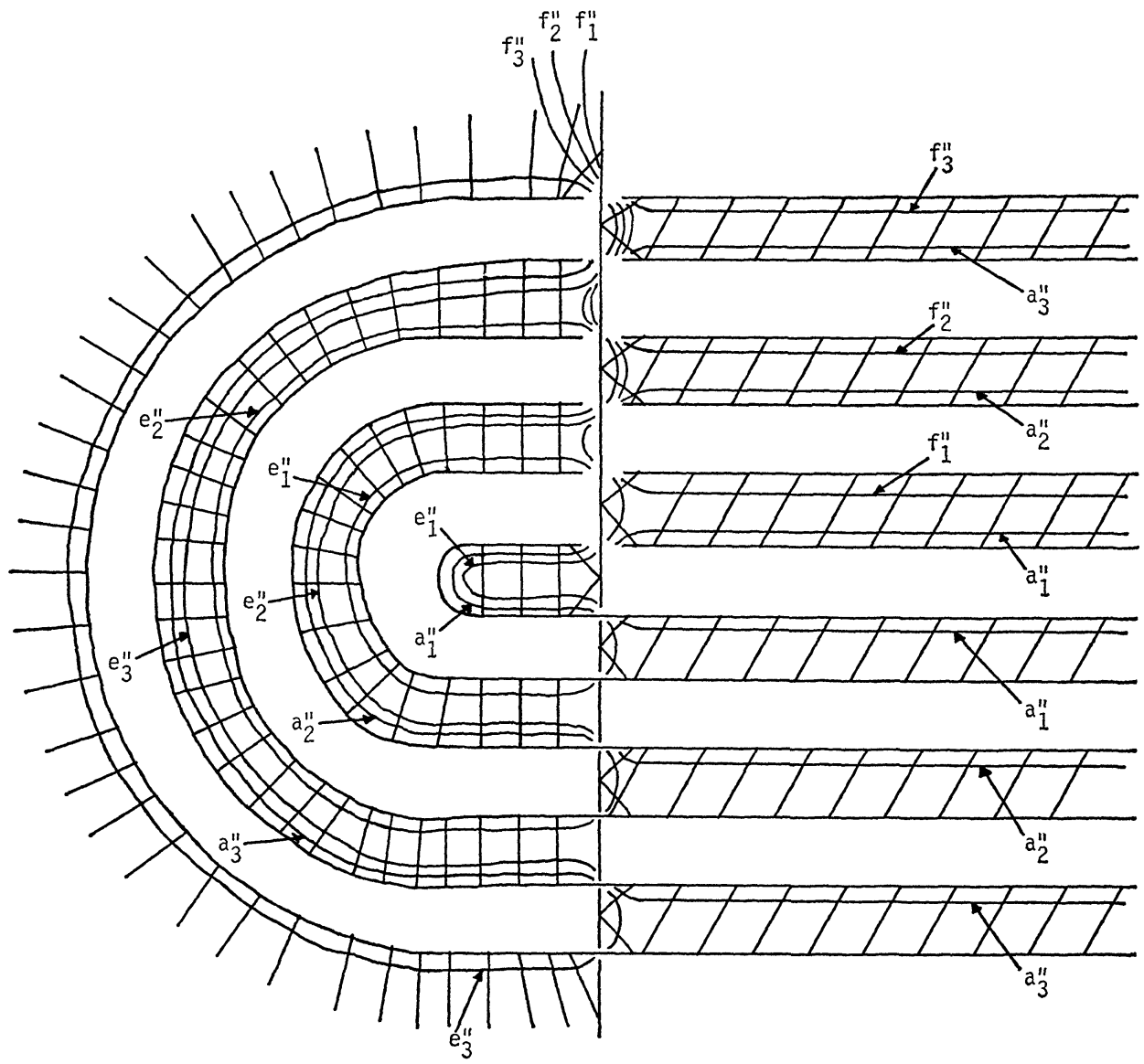

DIAGRAM 5

rechoosing the $n_{i}$. This completes the proof.

\section{REFERENCES}

1. S. Akbulut, On 2-dimensional homology classes of 4-manifolds, (preprint).

2. S. Akbulut and R. Kirby (private communication).

3. C. McA. Gordon, Knots, homology spheres and contractible 4-manifolds, Topology, 14 \#2 (1975), 151-172.

4. R. C. Kirby, ed., Problems in Low Dimensional Manifold Theory, Proc. AMS Summer Inst. in Top.-Stanford (1976).

5. A. G. Tristram, Some cobordism invariants for links, Proc. Camb. Phil. Soc., \#66(1969), 251-264.

Received January 8, 1979 and in revised form April 17, 1979.

UNIVERSTTY OF KENTUCKY

LEXINGTON, KY 40506 



\section{PACIFIC JOURNAL OF MATHEMATICS}

\section{EDITORS}

DONALD BABBITT (Managing Editor)

University of California

Los Angeles, California 90024

HUGO ROSSI

University of Utah

Salt Lake City, UT 84112

C. C. MOORE and ARTHur AGUS

University of California

Berkeley, CA 94720
J. DUGUNDJI

Department of Mathematics

University of Southern California

Los Angeles, California 90007

R. FINN and J. MILGRAM

Stanford University

Stanford, California 94305

\section{ASSOCIATE EDITORS}
R. Arnes
E. F. BECKENBACH
B. H. NeumanN
F. WOLF
K. YösHIDA

\section{SUPPORTING INSTITUTIONS}

UNIVERSITY OF ARIZONA

UNIVERSITY OF BRITISH COLUMBIA

CALIFORNIA INSTITUTE OF TECHNOLOGY

UNIVERSITY OF CALIFORNIA

MONTANA STATE UNIVERSITY

UNIVERSITY OF NEVADA, RENO

NEW MEXICO STATE UNIVERSITY

OREGON STATE UNIVERSITY
UNIVERSITY OF OREGON

UNIVERSITY OF SOUTHERN CALIFORNIA

STANFORD UNIVERSITY

UNIVERSITY OF HAWAII

UNIVERSITY OF TOKYO

UNIVERSITY OF UTAH

WASHINGTON STATE UNIVERSITY

UNIVERSITY OF WASHINGTON 


\section{Pacific Journal of Mathematics}

Vol. 102, No. $1 \quad$ January, 1982

S. Agou, Degré minimum des polynômes $f\left(\sum_{i=0}^{m} a_{i} X^{p^{r i}}\right)$ sur les corps finis

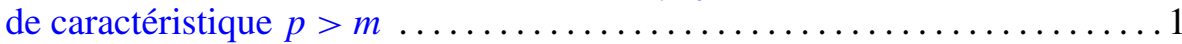

Chi Cheng Chen, On the image of the generalized Gauss map of a complete

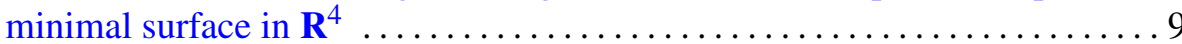

Thomas Curtis Craven and George Leslie Csordas, On the number of real

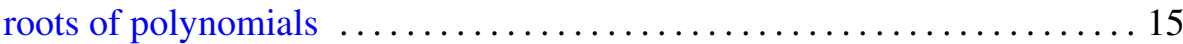

Allan L. Edelson and Kurt Kreith, Nonlinear relationships between oscillation and asymptotic behavior ....................... 29

B. Felzenszwalb and Antonio Giambruno, A commutativity theorem for

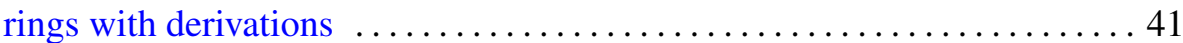

Richard Elam Heisey, Manifolds modelled on the direct limit of lines . . . . . 47

Steve J. Kaplan, Twisting to algebraically slice knots $\ldots \ldots \ldots \ldots \ldots \ldots 5$

Jeffrey C. Lagarias, Best simultaneous Diophantine approximations. II.

Behavior of consecutive best approximations $\ldots \ldots \ldots \ldots \ldots \ldots \ldots 61$

Masahiko Miyamoto, An affirmative answer to Glauberman's conjecture . . . 889

Thomas Bourque Muenzenberger, Raymond Earl Smithson and L. E.

Ward, Characterizations of arboroids and dendritic spaces ........... 107

William Leslie Pardon, The exact sequence of a localization for Witt

groups. II. Numerical invariants of odd-dimensional surgery

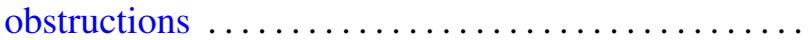

Bruce Eli Sagan, Bijective proofs of certain vector partition identities

Kichi-Suke Saito, Automorphisms and nonselfadjoint crossed products ...

John Joseph Sarraille, Module finiteness of low-dimensional PI rings ...

Gary Roy Spoar, Differentiable curves of cyclic order four . .

William Charles Waterhouse, Automorphisms of quotients of $\Pi \mathrm{GL}\left(n_{i}\right)$

Leslie Wilson, Mapgerms infinitely determined with respect to right-left equivalence

Rahman Mahmoud Younis, Interpolation in strongly logmodular

algebras 